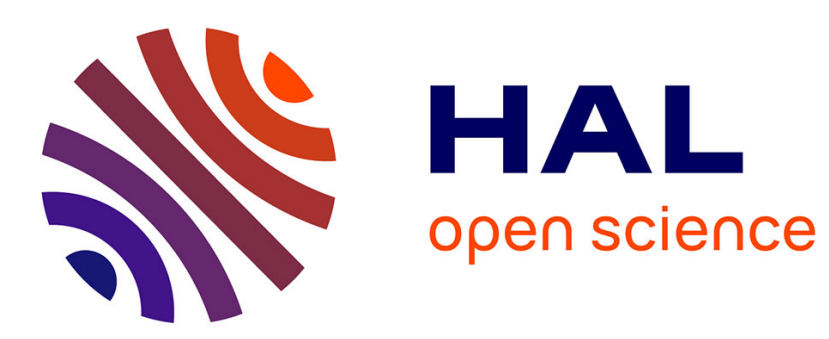

\title{
Multi-Scale Least-Weight Design of a Wing-Box via a Global/Local Approach
}

Enrico Panettieri, Marco Montemurro, Daniele Fanteria, Francesco Coccia

\section{To cite this version:}

Enrico Panettieri, Marco Montemurro, Daniele Fanteria, Francesco Coccia. Multi-Scale Least-Weight Design of a Wing-Box via a Global/Local Approach. Journal of Optimization Theory and Applications, 2020, 187, pp.776-799. 10.1007/s10957-020-01693-y . hal-02945365

\section{HAL Id: hal-02945365 https://hal.science/hal-02945365}

Submitted on 22 Sep 2020

HAL is a multi-disciplinary open access archive for the deposit and dissemination of scientific research documents, whether they are published or not. The documents may come from teaching and research institutions in France or abroad, or from public or private research centers.
L'archive ouverte pluridisciplinaire HAL, est destinée au dépôt et à la diffusion de documents scientifiques de niveau recherche, publiés ou non, émanant des établissements d'enseignement et de recherche français ou étrangers, des laboratoires publics ou privés. 


\title{
Multi-Scale Least-Weight Design of a Wing-Box via a Global/Local Approach
}

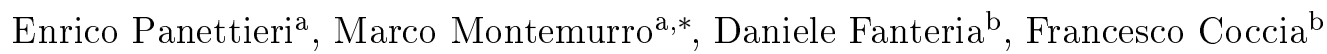 \\ ${ }^{a}$ Arts et Métiers Institute of Technology, Université de Bordeaux, CNRS, INRA, Bordeaux INP, HESAM \\ Université, I2M UMR 5295, F-33405 Talence, France \\ ${ }^{b}$ Universita di Pisa, Dipartimento di Ingegneria Civile e Industriale, Divisione Aerospaziale, Via \\ Girolamo Caruso, 8, 56122 Pisa, Italy
}

\begin{abstract}
In this work, a multi-scale optimization strategy for lightweight structures, based on a global-local modelling approach is presented. The approach is applied to a realistic wing structure of a civil aircraft. The preliminary design of the wing can be formulated as a constrained optimization problem, involving several requirements at the different scales of the structure. The proposed strategy is characterized by two main features. Firstly, the problem is formulated in the most general sense, by including all design variables involved at each problem scale. Secondly, two scales are considered: (i) the structure macroscopic scale, where low-fidelity numerical models are used; (ii) the structure mesoscopic scale (or component-level), where enhanced models are involved. In particular, the structural responses are evaluated at both global and local scales, avoiding the use of approximated analytical methods. To this end, fully parametric global and local finite element models are interfaced with an in-house genetic algorithm. Refined models are created only for the most critical regions of the structure, and linked to the global one by means of a dedicated sub-modelling approach.
\end{abstract}

Keywords: Optimization, Genetic algorithms, Wing, Stiffened panels, Global/local modelling approach

This is a pre-print of an article published in Journal of Optimization Theory and Applications.

The final authenticated version is available online at:

https://doi.org/10.1007/s10957-020-01693-y

\footnotetext{
${ }^{*}$ Corresponding author.

Email address: marco.montemurro@ensam.eu; marco.montemurro@u-bordeaux.fr (Marco Montemurro )
} 


\section{Introduction}

Mass reduction is a major concern in aircraft design. For this reason, the aircraft architecture is mostly made of thin-walled components, whose design is formulated as a constrained non-linear programming problem (CNLPP). The merit function is the structural mass and the CNLPP includes a set of design requirements formulated as constraints. Semi-monocoque structures are widely employed for fuselage and wing architectures, due to their high specific stiffness and specific strength. This type of structure is often referred as lightweight structure (LS). Of course, the design criteria for LSs include both local phenomena (i.e. at the scale of the single component, like a stiffened panel) and global ones (i.e. at the scale of the whole structure). Accordingly, a multi-scale modelling approach (MSMA) is needed to catch the coupling effect between the physical phenomena, intervening at different scales. Therefore, a dedicated multi-scale optimization (MSO) strategy, integrating a MSMA, is of paramount importance for LSs.

The preliminary phase of aircraft structural design is mostly based on analytical or semi-empirical models, developed since 40s [1-3]. These design methodologies have been continuously improved during the years, until becoming an established reference for aircraft designers $[4,5]$. However, these approaches rely on many simplifying hypotheses. For example, when dealing with the wing-box design, at the macroscopic scale, a rectangular cross-section is considered with simplified boundary conditions (BCs). Moreover, an equivalent thickness is considered for the skin, which takes into account also for the presence of the stringers. At the stiffened panel scale (mesoscopic scale), plane plates with uniformly loaded edges and (idealized) periodic BCs are considered for the buckling factor assessment; the stringers are considered as isolated and the Euler column buckling equations are used [4]. Preliminary design methodologies for the wing-box structure, integrating the aforementioned analytical models into iterative procedures, have been widely used in the literature [6]. The solution search is often performed through a deterministic algorithm: the initial guess is generally set by means of handbook rules [4]. Often, the number of design variables is reduced by introducing simplifying hypotheses or empirical rules. Of course, this approach extremely shrinks the design domain.

Several works on improved analytical or numerical methods for predicting the mechanical response of stiffened panels, especially regarding the buckling and post-buckling behaviour of such components [7,8] can be found in the literature. However, the aforementioned simplifying hypotheses are still used.

In 1972, Sobieszczanski and Loendorf [9] proposed a mixed optimization method for an aircraft fuselage, in which a lumped global finite element (FE) model, denoted as GFEM, was used (instead of analytical formulae) to evaluate the stiffness of the fuselage to obtain a better approximation of the loads, which were used for assessing the buckling factor of the stiffened panels by means of analytical formulae. Similarly, Fischer et al. [10] proposed a multi-level framework for the optimization of LSs, wherein a simplified GFEM is used to assess the average membrane and bending loads for the local optimization of panels performed using VICONOPT, a program based on analytical solutions for prismatic plane panels with simply supported edges and/or periodic BCs.

In all the aforementioned works the local analysis (and the related optimization) is performed by considering fixed internal loads resulting from the GFEM, hence neglecting stress redistribution due to the change of geometry at the upper scale, i.e. that of the whole structure. Therefore, the main limitation of such design procedures is related to a poor evaluation of the mechanical response of the structure, due either to the use of simplified models or to the approximation of the BCs in the passage from the GFEM to the local FE model (LFEM). In order to overcome these limitations, the utilisation of a proper 
global/local (GL) modelling strategy in the framework of the MSO of LSs is proposed in this work. GL modelling approaches allow the assessment of phenomena involved at the component-level through the use of LFEMs with realistic BCs derived from the GFEM. Both models have affordable computational costs, thus they can be integrated into an optimization strategy [11]. GL modelling approaches have been investigated mainly during 80 s and 90s [12-16]. However, as dicussed in [17], the vast majority of studies dealing with the structural analysis of wing-box architectures, for preliminary design purposes, do not integrate GL approaches $[18,19]$. This is manly due to the important computational effort required to perform scale transition between global and local models, which is often not compatible with the overall time required to perform the optimization process [20].

As far as the development of GL strategies for the structural analysis of the aircraft lifting system is concerned, some works can be found in the literature. In [21], a GL strategy dedicated to Damage Tolerance Analysis (DTA) of conventional wings is presented. Of course, the DTA needs a refined LFEM of the structural components in order to simulate the crack growth.

A more complete GL modelling approach for an aircraft wing is presented in [22]. The GFEM does not take into account for stringers and spar-caps, since stiffened panels are modelled as equivalent shells. Furthermore, only continuous variables are considered, which allows authors to use deterministic algorithms for the solution search. In [23], a GL approach for a high-speed wing is presented. The main issue is that LFEMs are mapped to rectangular plane stiffened plates, loosing, thus, the effects of the real geometry on instability phenomena. Furthermore, several constraints are evaluated using analytical formula. More recently, GL modelling framework for optimization of curvilinear spars and ribs (SpaRibs) has been presented in [24]. However, the resolution of the related CNLPP requires a huge computational cost: the proposed GL approach needs hundreds of software licences and hundreds of cores to find solutions in a reasonable time.

As it can be inferred from this (non-exhaustive) literature survey, GL modelling strategies are mainly used in the structural analysis of the wing-box structure of conventional aircraft. However, such strategies are rarely coupled to optimization methods due to three main issues: (a) the high computational costs related to the GL modelling approach; (b) the lack of pertinent criteria to automatically identify the zones of interest (ZOIs) within the GFEM; (c) the lack of suitable modelling strategies to automatically generate the LFEMs, by extracting pertinent information from the GFEM and by taking into account for variable geometry and mesh.

In this scenario, a MSO approach, integrating an efficient GL modelling strategy to evaluate all the necessary structural responses (at each pertinent scale) is presented in this work. The resulting optimization strategy is denoted as GL-MSO strategy. The GL modelling approach used in this study belongs to the family of the so-called submodelling GL methods sub-modelling $[13,14]$. In the usual work-flow of sub-modelling GL approaches, firstly a low fidelity linear analysis on a GFEM with a coarse mesh is run to identify one or more zones of interest (ZOIs). Then a refined LFEM is created for each ZOI and the analysis is performed by imposing displacements provided by the GFEM as BCs. Moreover, iterative stages can be added if the stress redistribution due to local effects is considered non-negligible.

The effectiveness of the GL-MSO strategy is proven on a meaningful real-world engineering problem: the least-weight design of a wing-box section of a wide-body aircraft that undergoes multiple loading conditions and subject to constraints of different nature. The physical responses, evaluated by means of both GFEM and LFEMs, are integrated into the CNLPP formulation as optimization constraints. The exchange between GFEM 
and LFEMs and the extraction of LFEMs from the GFEM are realised in a completely automatic way, without the user's intervention. The solution search for the CNLPP at hand is carried out by means of the ERASMUS (EvolutionaRy Algorithm for optimiSation of ModUlar Systems) algorithm developed by Montemurro [25], which is a special genetic algorithm (GA) able to deal with CNLPPs characterized by a variable number of design variables. This GA has been successfully used in other real-world engineering problems [26-34].

The paper is organised as follows. A general description of the design problem, the underlying hypotheses and the driving design criteria is given in Sec. 2. The mathematical formulation of the multi-scale design problem and the adopted numerical strategy are discussed in Sec. 3. The details of the GL modelling approach are presented in Sec. 4, whilst numerical results are shown in Sec. 5. Finally, Sec. 6 ends the paper with some conclusions and prospects.

\section{Multi-Scale Least-Weight Design of a Metallic Wing: Problem Description}

The wing-box considered in this study is representative of the wing structure of a short/medium-range, narrow body, commercial passenger twin-engine jet airliner. Because of the symmetry of the structure with respect to the $x_{b}-z_{b}$ plane, as shown in Fig. 1, and the loading conditions, only half-wing has been considered.

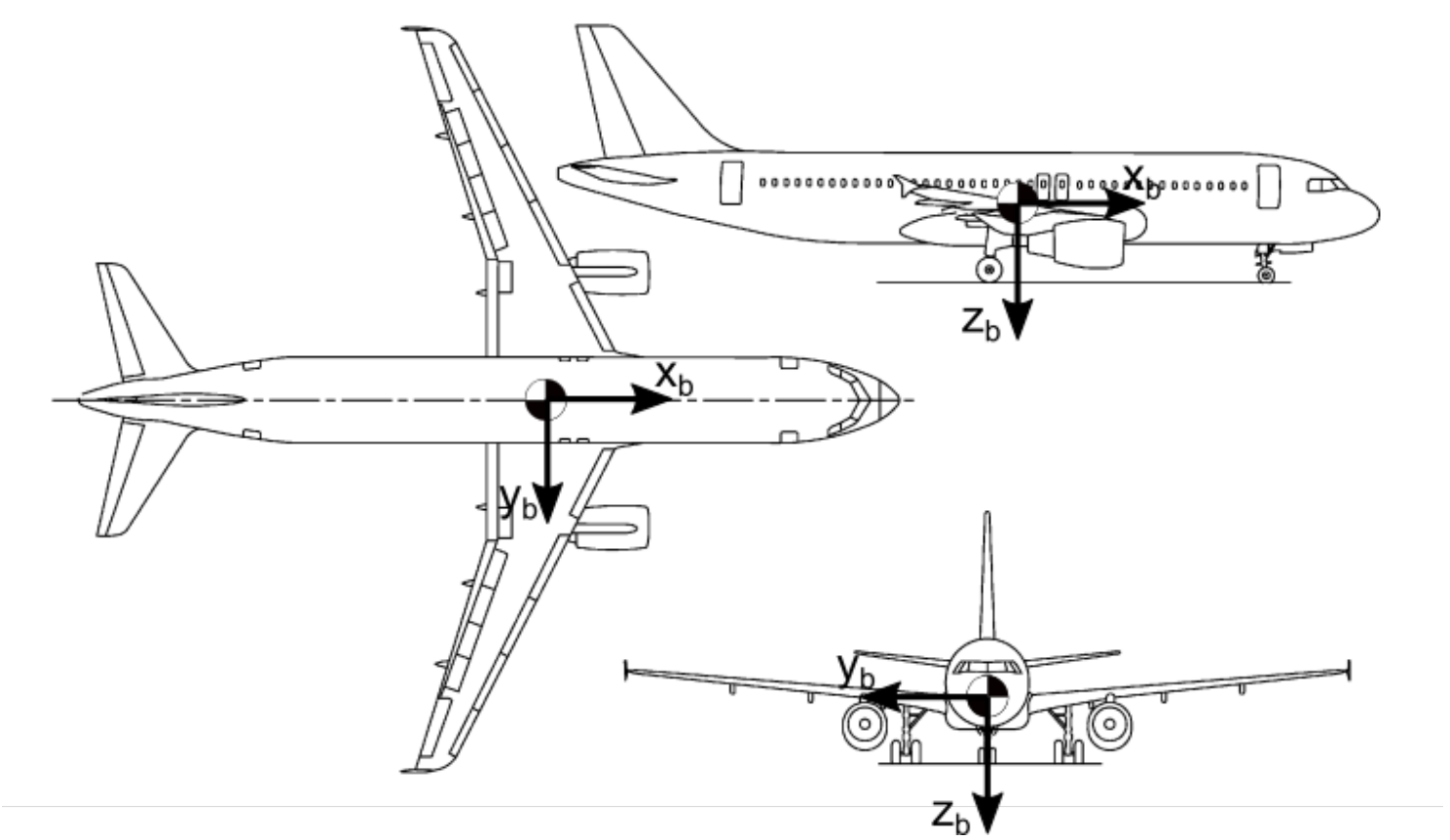

Figure 1: Example of a twin-engine jet airliner together with the global body reference centered in the aircraft center of gravity (adapted from [35]).

\subsection{Wing-Box Geometry and Material Properties}

The wing-box external geometry is defined with respect to the leading edge of the wing through three relevant sections, i.e. root, kink and tip section. The wing planform ( $s$ is the span-wise wing coordinate), the wing-box spar positions and the normalized shape of the supercritical airfoil profile F15-11 are shown in Fig. 2. In order to take into account for the dihedral angle and the twist angle, the airfoil profile is suitably scaled and 
rotated at the three wing-box sections parallel to the free-stream direction. To generate the wing-box external shape between the sections at the root, at the kink and at the tip, a linear interpolation is used. The relevant parameters used to model the wing-box external geometry are reported in Table 1 .

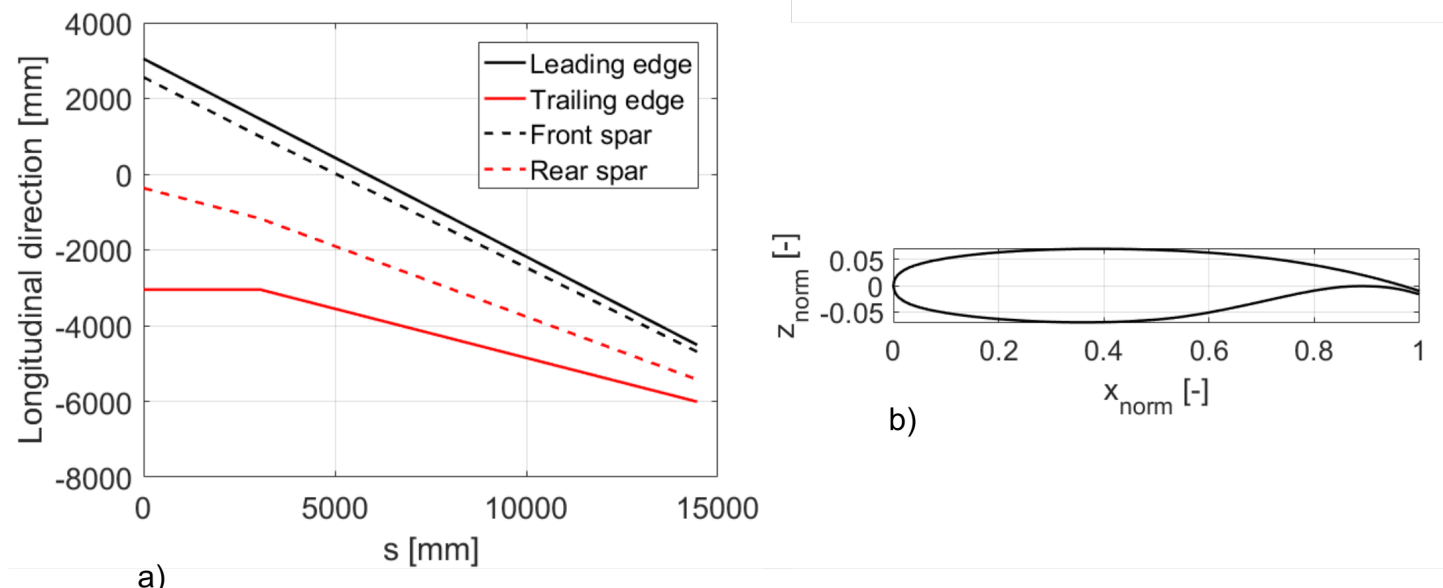

Figure 2: (a) Wing planform: position of leading edge, trailing edge, front spar and rear spar. (b) Normalized airfoil profile F15-11.

Table 1: Main geometrical parameters of the wing-box structure.

\begin{tabular}{|c|c|c|c|c|}
\hline \multicolumn{5}{|c|}{ Wing global parameters } \\
\hline & \multicolumn{2}{|c|}{ Leading edge sweep angle [deg] } & \multicolumn{2}{|l|}{27.6} \\
\hline & \multicolumn{2}{|r|}{ Diehdral angle [deg] } & \multicolumn{2}{|l|}{5.1} \\
\hline \multicolumn{5}{|c|}{ Wing span-wise parameters } \\
\hline & & Root & Kink & Tip \\
\hline Span coordinate, $s$ & [mm] & 0 & 3059 & 14459 \\
\hline Chord length, $c$ & [mm] & 6100 & 4308 & 1500 \\
\hline Twist, $\theta$ & [deg] & 3.7 & 3.5 & -2.0 \\
\hline Front spar position & {$[-]$} & $8 \% c_{\text {root }}$ & $10.75 \% c_{\text {kink }}$ & $12 \% c_{\mathrm{tip}}$ \\
\hline Rear spar position & {$[-]$} & $56 \% c_{\text {root }}$ & $61 \% c_{\text {kink }}$ & $61 \% c_{\text {tip }}$ \\
\hline Thickness profile & [mm] & 920 & 440 & 160 \\
\hline
\end{tabular}

Some simplifications are introduced in the modeling of the wing-box structural components:

a. only main structural components are modeled, i.e. skin, stringers, ribs, spar and spar caps

b. perfect bonding is assumed between the modeled components

c. cut-outs and openings are not considered at this design stage. 
The ribs between the root section and the kink one (in-board wing) are parallel to the free stream direction, while those between kink and tip section (out-board wing) are perpendicular to the out-board rear spar. Eventually, the tip rib orientation is parallel to the free stream direction. To introduce a gradual change between the orientations of the in-board and of the out-board ribs as well as between the out-board ribs and the tip rib, two transition ribs have been introduced as shown in Fig. 3 a).

Stringers are created at the intersection of planes perpendicular to the top and bottom skins and parallel to the out-board rear spar. Stringers have a T-shaped cross-section, while spar-caps are characterized by an L-shaped cross-section. Since stringers run-outs are not modeled at this design stage, stringers do not intersect the front or the rear spar in order to avoid the stiffening of the wing-box structure. All these geometrical details are illustrated in Fig. 3.

All structural elements are made of a 2024-T3 aluminium alloy, whose behaviour is as-
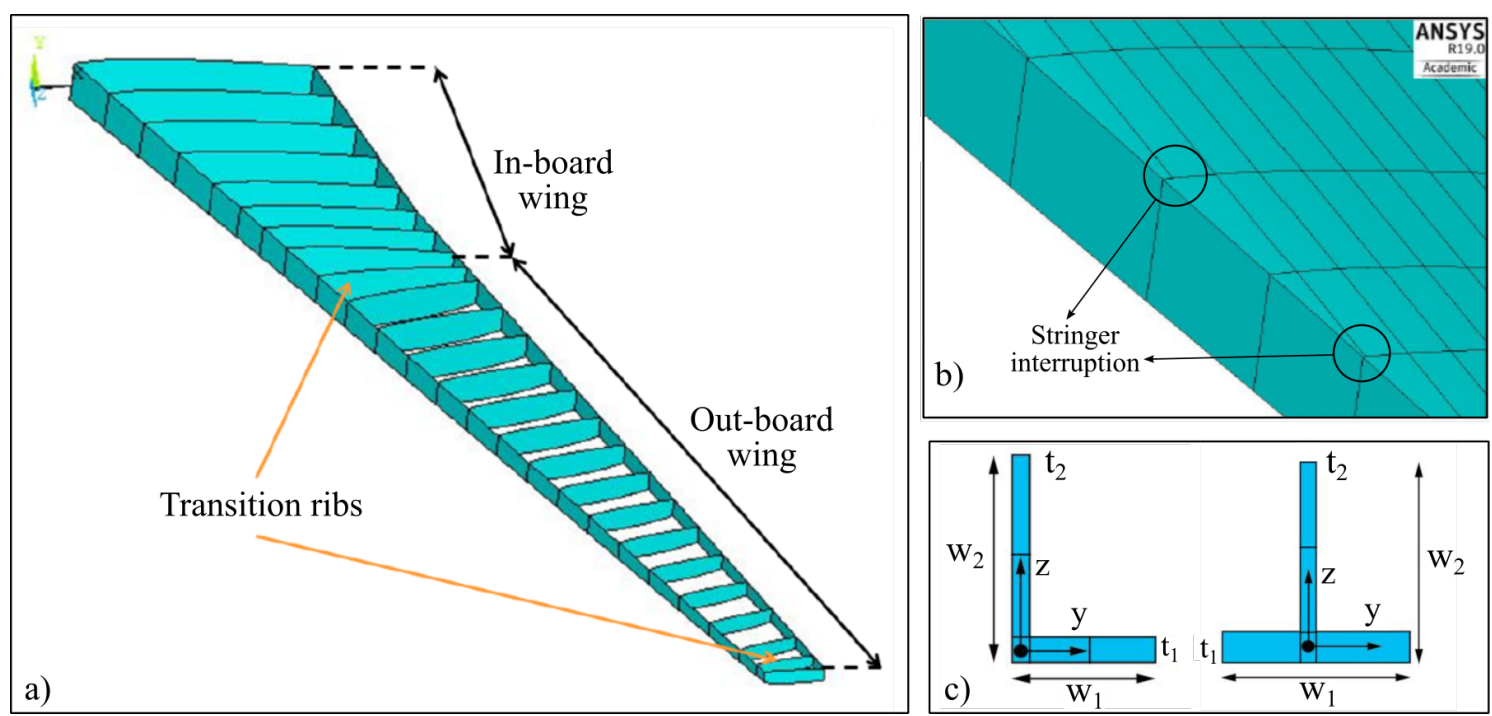

Figure 3: a) Ribs orientations in the in-board and out-board wing regions; b) example of a stringer interruption before the front spar; c) geometrical parameters of the spar-cap cross-section (left) and of the stringer cross-section (right).

sumed to be linear elastic homogeneous isotropic: the relevant material properties are listed in Table 2.

Table 2: Material properties of the 2024-T3 aluminium alloy.

\begin{tabular}{cc|c|cc|c}
\hline Young's modulus, E & {$[\mathrm{MPa}]$} & 0 & Poisson's ratio, $\nu$ & {$[-]$} & 0.3 \\
\hline Yield strength, YS & {$[\mathrm{MPa}]$} & 340 & Ultimate strength, US & {$[\mathrm{MPa}]$} & 400 \\
\hline Density, $\rho$ & {$\left[\mathrm{kg} / \mathrm{m}^{3}\right]$} & 2780 & & & \\
\hline
\end{tabular}

\subsection{Design criteria}

The design criteria to be used for the optimization of the wing-box structure should ponder several loading conditions and mechanical phenomena that the structure could encounter during the operative life [36]. 
Criteria related to fatigue or aeroelastic loads can also play an important role within the design process of aircraft structures. Fatigue loads can be taken into account by either a durability approach, aiming at the full component operability over the entire operational life, or a damage tolerance approach, which must ensure the component to be operative between the inspection intervals. In this work, the durability approach is adopted by means of an equivalent static check.

Aeroelastic design criteria have not been considered in the preliminary design phase (as usual in the case of wing-box structures).

The following set of design criteria (DC) is integrated in the formulation of the multi-scale optimization problem:

- DC1: the maximum vertical tip displacement, $\delta_{\text {tip }}$, must be lower than a reference value, $\delta_{\text {tip }}^{\text {ref }}$.

- DC2: the maximum torsional twist, $\theta_{\text {tip }}$, at the wing-box tip must be lower than a reference value, $\theta_{\text {tip }}^{\text {ref }}$.

- DC3: for an appropriate Ground-Air-Ground (GAG) load spectrum, no fatigue failure must occur.

- DC4: a no-buckling design is sought for both top wing and bottom wing.

- DC5: the ribs must be characterized by a suitable compressive stiffness in order to prevent warping.

DC1 and DC2 represent a check on the vertical and torsional stiffness of the wing-box structure. DC3 represents an equivalent static check expressed in terms of the detail fatigue ratio (DFR) method [37], for which an allowable stress value of $250 \mathrm{MPa}$ is obtained. DC4 is enforced to a sub-domain of all the panels forming both the top and the bottom wing. Critical stiffened panels are identified through a pertinent selection criterion in order to obtain a no-buckling structure (the reference buckling factor, $\lambda_{\text {ref }}$ is set equal to 1.6). The assessment of DC4 requires a coupling between the GFEM of the wing-box and the LFEM of the most critical stiffened panels, as discussed in Sec. 4.1. DC5 is included within the formulation in terms of a constraint on the rib thickness to prevent ribs from warping. More details regarding the above DC are given in Sec. 3.

\subsection{Load cases}

During the wing-box preliminary design phase, several loading conditions should be included into the problem formulation in order to assess the mechanical response of the structure under positive and negative maneuvers, dynamic and static gusts, aileron roll and taxiing. However, since the aim of this study is to highlight the effectiveness of the GL modelling strategy in the context of the GL-MSO approach, only two loading cases (LCs) have been considered under limit load (LL) condition for a maximum take-off mass, $W_{\max }^{\mathrm{TO}}$, equal to $73474 \mathrm{~kg}$ :

- LC1 (up-bending case): the symmetric pull-up manoeuvre at diving speed, $V_{D}$ as shown in Fig.4 (left) and a vertical loading factor, $n_{z}=2.5$ have been considered.

- LC2 (down-bending case): the inverted flight condition at the design maneuvering speed, $V_{H}$ of Fig.4 (left), and a vertical loading factor, $n_{z}=-1$ have been considered.

For these LCs, four types of loads have been applied to the wing-box structure:

a. aerodynamic loads; 
b. fuel weight distribution;

c. engine concentrated weight;

d. landing gear weight.

An estimation of the aerodynamic loads has been obtained by applying the Diederich method [38]. The wing loading distribution of five points of the maneuver envelope shown in Fig.4 (a) is reported in Fig.4 (b). The highest values of total lift correspond to $1.889 \cdot 10^{6}$ $\mathrm{N}$ at $V_{D}$ and $n_{z}=2.5$ whilst the lowest one is $-7.111 \cdot 10^{5} \mathrm{~N}$ at $V_{H}$ and $n_{z}=-1$.

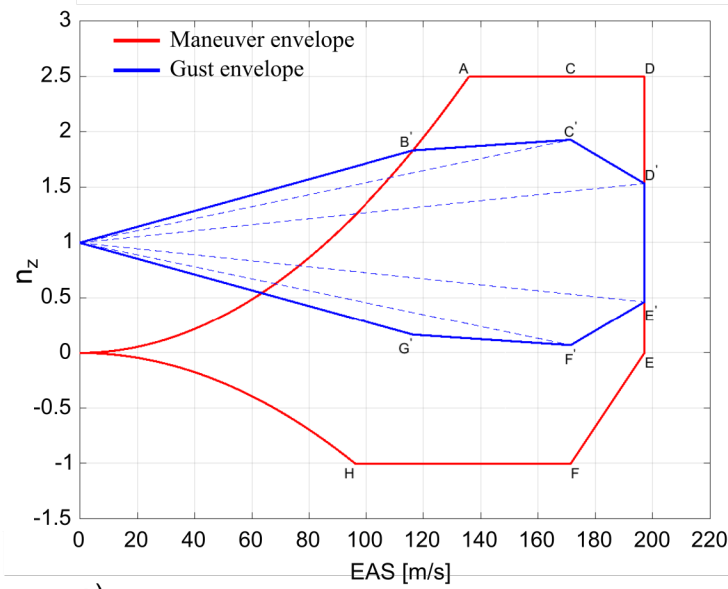

a)

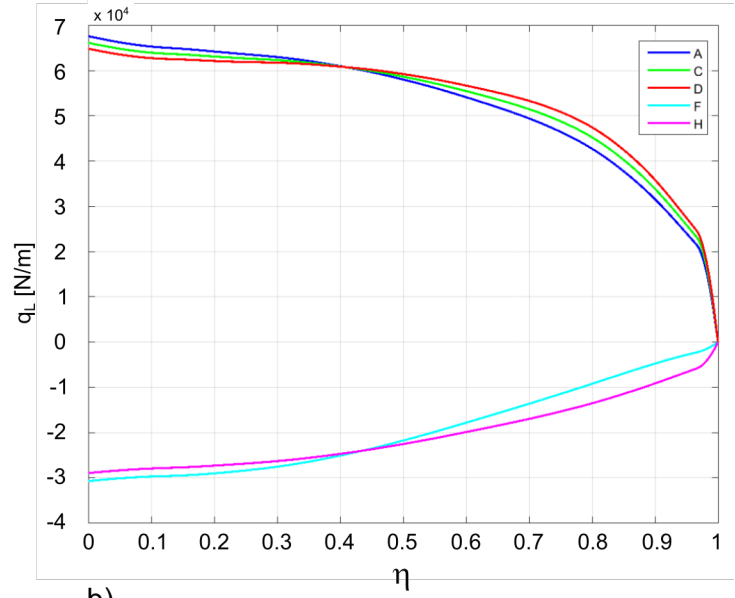

b)

Figure 4: (a) Maneuver and gust envelope obtained for $W_{\max }^{\mathrm{TO}}$, sea level altitude and retracted flaps. (b) Wing loading distribution for five characteristic points of the maneuver envelope.

The fuel weight distribution has been estimated for the steady flight condition at $W_{\max }^{\mathrm{TO}}$ with a fuel density, $\rho_{\text {fuel }}$ of $802 \mathrm{~kg} / \mathrm{m}^{3}$. Fig. 5 shows the values of the tank section, $A_{\text {tank }}$ and the fuel weight distribution, $q_{\text {fuel }}$, in function of the span-wise coordinate $s$.

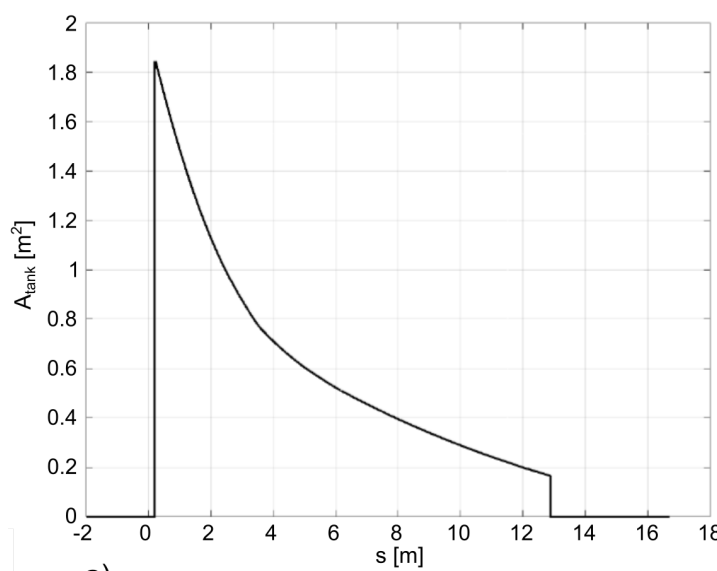

a)

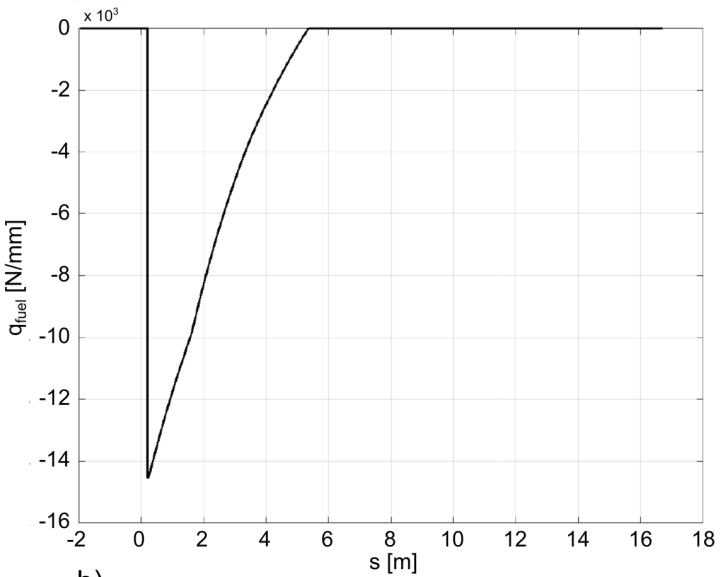

b)

Figure 5: Span-wise fuel tank section area distribution (a) and span-wise fuel weight distribution (b).

The CFM56-5B4 engine is installed at the wing-box kink section. Its weight and the pitching moment has been evaluated by considering a total mass, $W_{\text {eng }}=3629.8 \mathrm{~kg}$ and a value of eccentricity of the center of gravity of the engine of $3 \mathrm{~m}$. Eventually, the weight of 
the landing gear has been included as an additional concentrated load on two of the closest ribs to the span-wise wing coordinate $s=2 \mathrm{~m}$. The value of the landing gear mass is 950 $\mathrm{kg}$.

Due to the presence of the aerodynamic loads, the fuel, the engine and the landing gear weights, the two LCs modify significantly the span-wise wing load distribution since during the the pull-up manoeuvre the wing is bended upward, whilst the weight of the engine, of the landing gear and of the fuel point downward. Conversely, in the inverted flight condition, the wing is bended downward while the engine, the landing gear and the fuel loads point upward. The shear load span-wise distribution as well as the bending and the pitching moments for both LCs, are shown in Figs. 6 and 7, respectively.

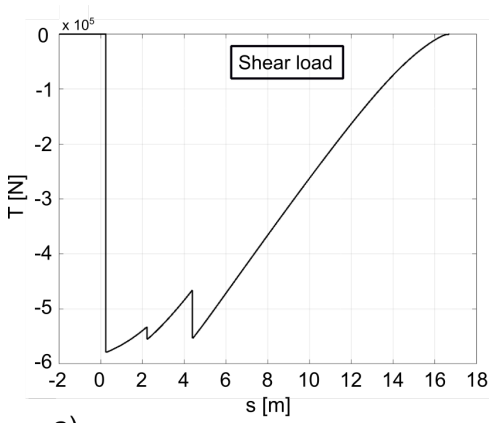

a)

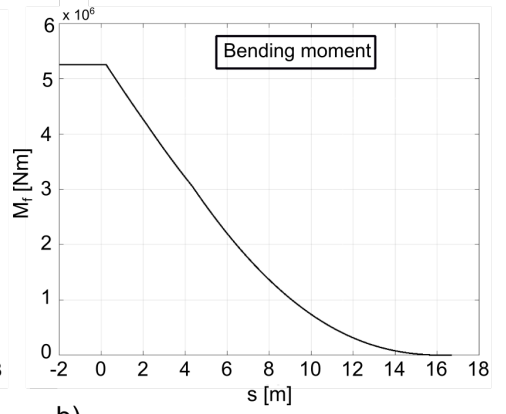

b)

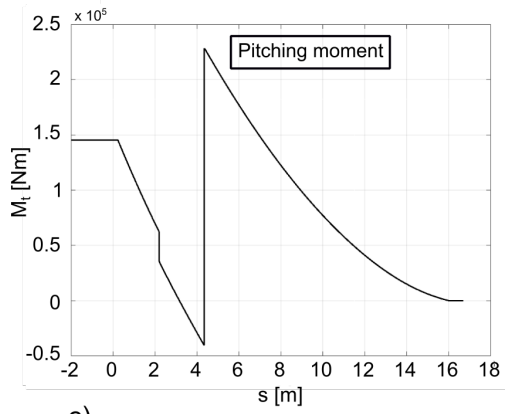

c)

Figure 6: (a) Shear load, (b) bending moment and (c) pitching moment span-wise distribution of the symmetric pull-up manoeuvre (LC1).
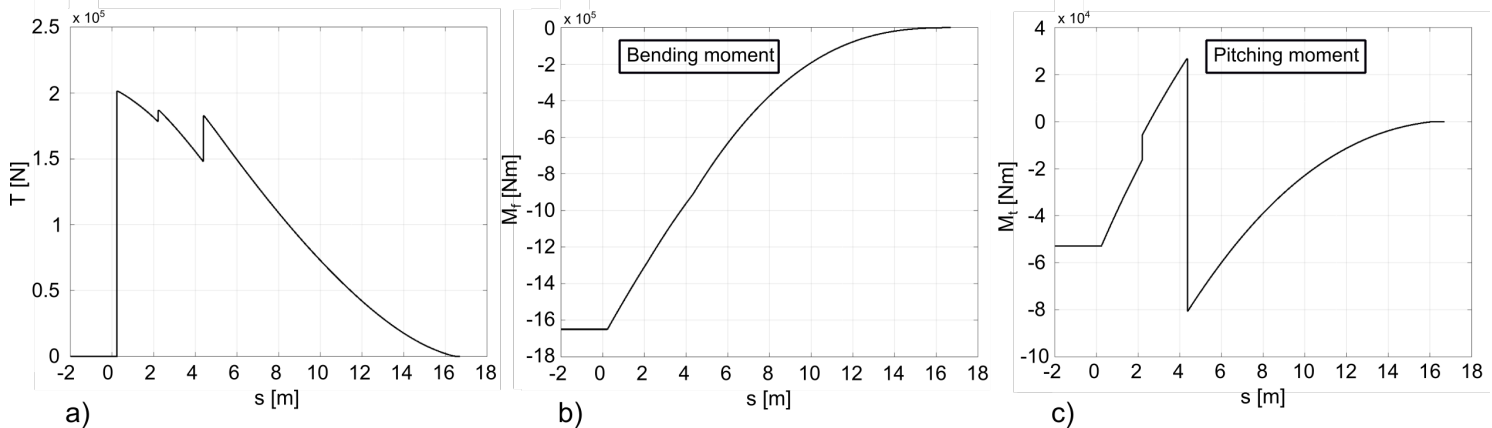

Figure 7: (a) Shear load, (b) bending moment and (c) pitching moment span-wise distribution of the inverted flight condition (LC2).

\section{Mathematical Formulation of the Optimization Problem}

\subsection{Design Variables}

In this study, the design variables modify the geometrical properties of the internal structural components of the wing-box such as: skin, stringer, spar, spar cap and rib.. Conversely, the wetted area and the position of the wing-box with respect to the fuselage is set a-priori. Moreover, the structural components located at the ventral and dorsal regions of the wing-box are characterized by different geometrical variables in order to fulfil the different design requirements.

Scaling parameters have been introduced in order to modify the geometrical properties in the span-wise direction. In particular, linear piecewise functions have been defined between 
the root, the kink and the tip span-wise coordinates as shown in Fig. 8, which illustrates the variation of the generic geometrical parameter, $\xi(s)$ in both inboard and outboard sections.

For each function, four parameters are defined: two slopes $\left(m_{\text {in }}\right.$ and $\left.m_{\text {out }}\right)$ and two amplification factors $\left(\alpha_{\text {in }}\right.$ and $\left.\alpha_{\text {out }}\right)$ according to the following formulae:

$$
\begin{aligned}
& m_{\text {in }}=\frac{\xi\left(s_{\text {kink }}\right)-\xi\left(s_{\text {root }}\right)}{s_{\text {kink }}-s_{\text {root }}}, m_{\text {out }}=\frac{\xi\left(s_{\text {tip }}\right)-\xi\left(s_{\text {kink }}\right)}{s_{\text {tip }}-s_{\text {kink }}}, \\
& \alpha_{\text {in }}=\frac{\xi\left(s_{\text {root }}\right)}{\xi\left(s_{\text {kink }}\right)}, \alpha_{\text {out }}=\frac{\xi\left(s_{\text {kink }}\right)}{\xi\left(s_{\text {tip }}\right)}, \quad \text { with } \alpha_{\text {in }}>1 \text { and } \alpha_{\text {out }}>1 .
\end{aligned}
$$

Eventually, continuity conditions are imposed to ensure the equivalence of the value of the generic geometrical property at the kink section.

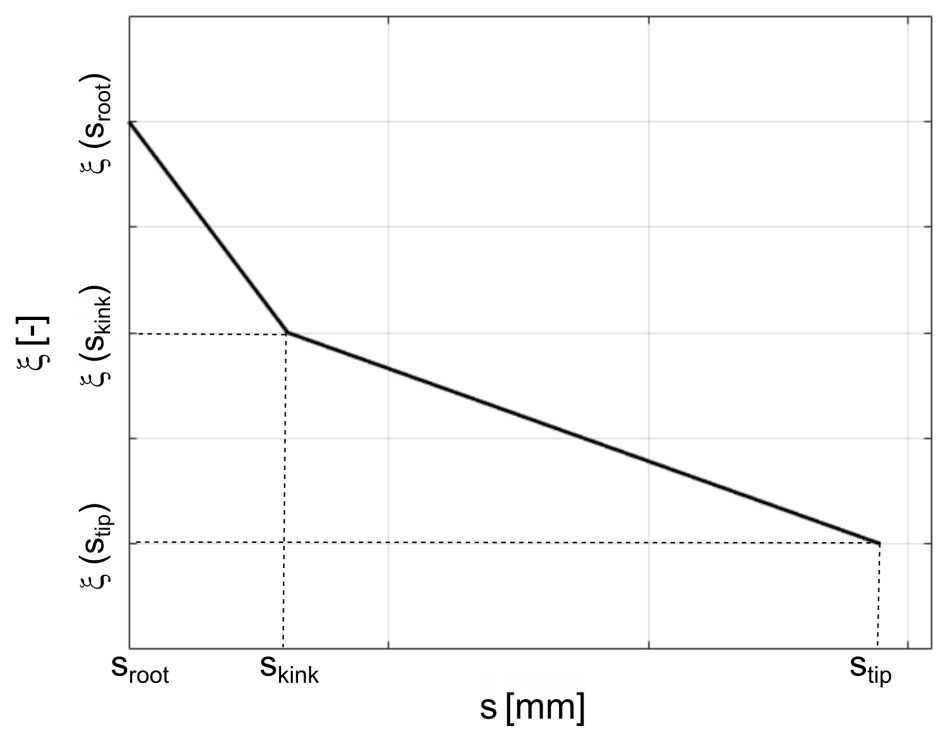

Figure 8: Linear piecewise function used to scale the generic geometrical parameter, $\xi$.

The span-wise variation of $d$ can be written as:

$$
\xi(s)=\left\{\begin{array}{cc}
\xi\left(s_{\text {tip }}\right)\left[1+\frac{s_{\text {tip }}-s}{s_{\text {tip }}-s_{\text {kink }}}\left(\alpha_{\text {out }}-1\right)\right] & \text { with } s_{\text {kink }}<s<s_{\text {tip }}, \\
\alpha_{\text {out }} * \xi\left(s_{\text {tip }}\right)\left[1+\frac{s_{\text {kink }}-s}{s_{\text {kink }}-s_{\text {root }}}\left(\alpha_{\text {in }}-1\right)\right] & \text { with } s_{\text {root }}<s<s_{\text {kink }} .
\end{array}\right.
$$

According to Eq. (3), every geometrical quantity is defined with respect to its value at the wing tip.

The design variables defining each structural component are briefly described in the following.

Skins. Two sets of design variables are defined for the dorsal skin and the ventral one: the values of thickness at the tip sections, $t_{\text {skin-tip }}^{\text {top }}$ and $t_{\text {skin-tip }}^{\text {bot }}$, as well as the values of the amplification factors, $\alpha_{\text {skin-in }}^{\text {top }}, \alpha_{\text {skin-out }}^{\text {top }}, \alpha_{\text {skin-in }}^{\text {bot }}$ and $\alpha_{\text {skin-out }}^{\text {bot }}$.

The lower bound chosen for the wing tip thickness of the skin has been chosen equal to 
$2.7 \mathrm{~mm}$, which is a value commonly assumed for lightning strike requirements, while the upper bound value has been set as the $150 \%$ of the lower bound one.

Spars. The design variables used to define front and rear spars is the spar thickness at the wing tip with the corresponding amplification factors, $t_{\mathrm{spar}-\mathrm{tip}}, \alpha_{\mathrm{spar}-\mathrm{in}}$ and $\alpha_{\mathrm{spar}-\mathrm{out}}$. In this work, for the sake of simplicity, no holes or stiffening beads are considered in the spar model. For this component, a lower bound value of $1.2 \mathrm{~mm}$, as a minimum machinability thickness, has been used. The upper value of the variable has been set equal to that of the skin.

Ribs. The value of the thickness of the rib at the tip of the wing and the amplification factors characterize the set of the design variables of the ribs, $t_{\text {rib-tip }}, \alpha_{\text {rib-in }}$ and $\alpha_{\text {rib-out }}$. Like the spars, no beads or holes are considered. Moreover, within this study the pitch of the ribs is set equal to $700 \mathrm{~mm}$.

Spar-caps. Spar-caps have an L-shaped cross-section (see Fig. 3). The design variables characterizing this component are the flange width and height (assumed identical) at the wing tip, $w_{\text {spar-tip }}$, the thickness of the flanges at the wing tip, $t_{1-\text { spar-tip }}$ and $t_{2-\text { spar-tip }}$, and the amplification factors, $\alpha_{\text {spar-in }}$ and $\alpha_{\text {spar-out }}$.

The lower bound of the flange width is $16 \mathrm{~mm}$, which is 4 times the minimum rivet diameter, whilst the minimum thickness of the flange attached to the skin is $2.1 \mathrm{~mm}$, which is 0.8 times the lower bound of the skin thickness. Conversely, the lower bound of the flange thickness attached to the spars has been set to $1.2 \mathrm{~mm}$, which represents the minimum machinability thickness.

Stringers. Two sets of design variables are defined for the stringers of both dorsal and ventral regions. Each set includes the parameters defining the T-shaped cross-section (see Fig. 3), i.e. the flange length, $w_{1}$, assumed identical to the web length, flange and web thickness, $t_{1}$ and $t_{2}$, respectively, the stringer pitch, $p_{\mathrm{str}}$, and a correction factor.

To ensure a minimum distance between stringers at the wing root (set equal to the $25 \%$ of the stringer pitch) and to avoid, at the same time, the superposition of the flanges, the amplification factors, $\alpha_{\text {in }}$ and $\alpha_{\text {out }}$, are computed as follows:

$$
\alpha_{\text {in }}=\sqrt{\frac{\beta}{\chi}}, \alpha_{\text {out }}=\sqrt{\beta \chi}, \text { with } \beta=\frac{w_{1-\text { str-root }}}{w_{1-\text { str-tip }}},
$$

where $w_{1-\text { str-root }}$ and $w_{1-\text { str-tip }}$ are the values of the flange length at the root and tip sections, respectively, whilst $\chi$ is the correction factor which is used as design variable. The design variables defining the stringer geometry are: $p_{\mathrm{str}}^{\mathrm{top}}, p_{\mathrm{str}}^{\mathrm{bot}}, w_{1-\mathrm{str}-\mathrm{tip}}^{\mathrm{top}}, w_{1-\mathrm{str}-\mathrm{root}}^{\mathrm{top}}$, $w_{1-\text { str-tip }}^{\text {bot }}, w_{1-\text { str-root }}^{\text {bot }}, t_{1-\text { str }}^{\text {top }}, t_{1-\text { str }}^{\text {bot }}, t_{2-\text { str }}^{\mathrm{top}}, t_{2-\text { str }}^{\text {bot }}, \chi_{\text {str }}^{\text {top }}$ and $\chi_{\text {str }}^{\text {bot }}$.

The lower and upper bounds of the pitch of the stringer has been set dqual to $100 \mathrm{~mm}$ and $160 \mathrm{~mm}$, respectively (for both dorsal and ventral regions), in order to include typical pitch values for different categories of transport aircraft.

The lower bound of the flange length is $32 \mathrm{~mm}$, which is eight times the minimal rivet diameter. The lower bound of the stringer thickness at wing tip is $2.1 \mathrm{~mm}$.

All the above design variables are grouped into the array, $\boldsymbol{\xi}$,

$$
\begin{array}{r}
\xi^{\mathrm{T}}=\left\{t_{\mathrm{skin}-\mathrm{tip}}^{i}, \alpha_{\mathrm{skin}-j}^{i}, t_{\mathrm{spar}-\mathrm{tip}}, \alpha_{\mathrm{spar}-j}, t_{\mathrm{rib}-\mathrm{tip}}, \alpha_{\mathrm{rib}-j, w_{\mathrm{spar}-\mathrm{tip}}}, t_{1-\mathrm{spar}-\mathrm{tip}},\right. \\
\left.t_{2-\mathrm{spar}-\mathrm{tip}}, \alpha_{\mathrm{spar}-j}, p_{\mathrm{str}}^{i}, w_{1-\mathrm{str}-\mathrm{tip}}^{i}, w_{1-\mathrm{str}-\mathrm{root}}^{i}, t_{1-\mathrm{str}}^{i}, t_{2-\mathrm{str}}^{i}, \chi_{\mathrm{str}}^{i}\right\},
\end{array}
$$

with $i=$ top, bottom and $j=$ in, out.

The design variables for the problem at hand, with the relative intervals, are listed in Table 3 . The total number of design variables is 29 . 


\subsection{Objective Function and Optimization Constraints}

The goal of the GL-MSO approach is the minimization of the wing-box mass, which constitutes the objective function of the multi-scale design problem, defined as:

$$
\Phi(\boldsymbol{\xi})=\sum_{e=1}^{N_{e}^{\mathrm{GFEM}}} V_{e}(\boldsymbol{\xi}) \rho_{e},
$$

where $V_{e}(\boldsymbol{\xi})$ and $\rho_{e}$ are the volume and the density of the $e$-th element, constituting the GFEM of the wing-box, respectively, while $N_{e}^{\mathrm{GFEM}}$ is the total number of elements.

Before introducing the optimization constraints (OCs), two entities of the sets need to be defined: the repetitive structural unit (RSU) and the check zone of the GFEM, i.e. $\Gamma_{\text {check }}$. The RSU represents the elementary unit of the structural model, i.e. a portion of a stringer with the adjacent skin elements centered between two consecutive ribs. The RSU does not include areas of the model neither in proximity of spars (stringer interruption) nor in proximity of the root section, transitions and tip ribs. $\Gamma_{\text {check }}$ represents a subdomain of the GFEM from which the zones of interest (ZOIs) are extracted. The ZOIs are those regions (i.e. pertinent sub-sets of $\Gamma_{\text {check }}$ ) of the model for which a detailed LFEM is built and analyzed after the analysis conducted on the GFEM (see Sec. 4 for more details). $\Gamma_{\text {check }}$ is constituted of skin and stringers belonging to top (dorsal) and bottom (ventral) regions of the wing-box architecture, from which those zones adjacent to spars, root section, transition and tip ribs are excluded. Fig. 9 shows the RSU as well as the sub-domain $\Gamma_{\text {check }}$ of the dorsal region of the wing-box. To correctly formulate the DC

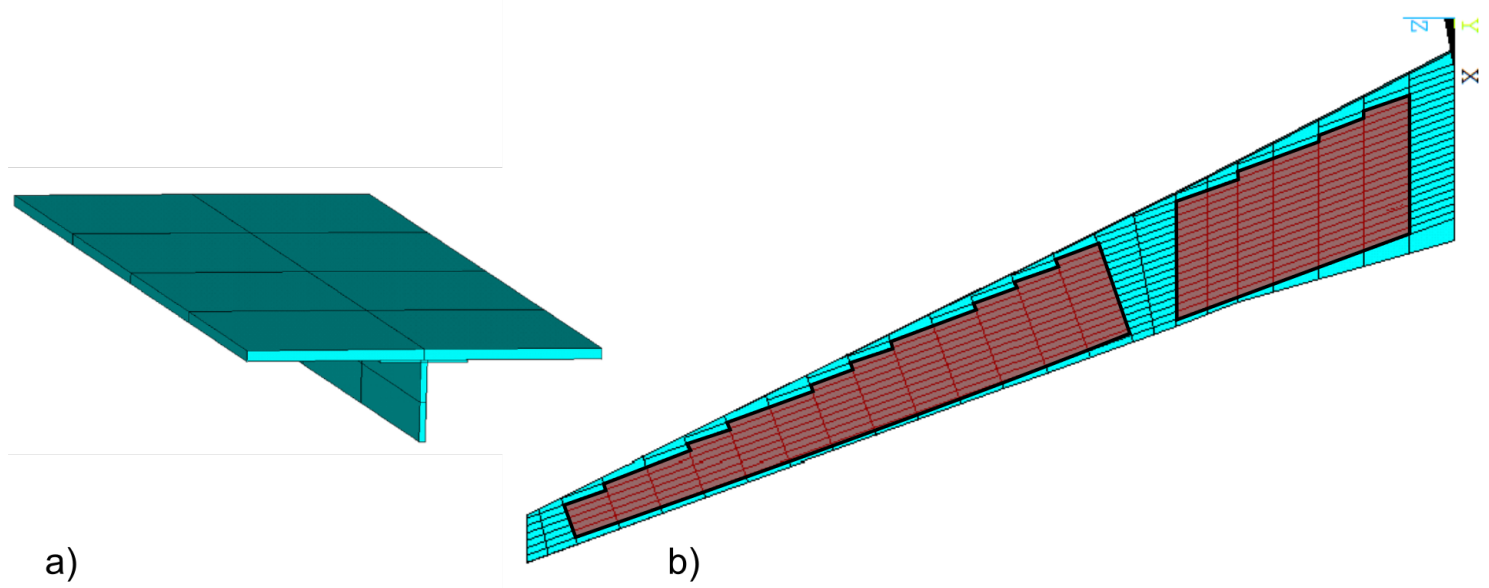

Figure 9: (a) The RSU with the beam section render activated and (b) the sub-domain $\Gamma_{\text {check }}$ for the dorsal region of the wing-box.

(see section 2.2) as OCs, the wing-box FE model, identified with $\Gamma_{\mathrm{GFEM}}$, is split into subregions: $\Gamma_{\text {skin }}^{i}, \Gamma_{\text {str }}^{i}, \Gamma_{\text {spar }}$ and $\Gamma_{\text {rib }}$ (with $i=$ top, bottom) refer to the combination of top and bottom skins, stringers, spars (with the spar caps) and ribs of the wing-box model, respectively. Consequently, the wing-box domain and the check zone read:

$$
\begin{gathered}
\Gamma_{\text {GFEM }}=\bigcup_{i=\text { bottom }}^{\text {top }}\left(\Gamma_{\text {skin }}^{i} \cup \Gamma_{\text {str }}^{i}\right) \cup \Gamma_{\text {spar }} \cup \Gamma_{\text {rib }}, \\
\Gamma_{\text {check }} \subset \bigcup_{i=\text { bottom }}^{\text {top }} \Gamma_{\text {skin }}^{i} \cup \Gamma_{\text {str. }}^{i} .
\end{gathered}
$$


Through this notation, the union of the ZOIs, referred as $\Gamma_{\text {LFEM }}$, represents the set of the most critical regions of the GFEM extracted from the check zone, i.e. $\Gamma_{\text {LFEM }} \subset \Gamma_{\text {check }}$. The first constraint function $(\mathrm{OC} 1)$ is related to $\mathrm{DC} 1$ and reads:

$$
h_{1}:=\max _{k} \frac{\left|\delta_{\text {tip }}\right|}{\delta_{\text {tip }}^{\text {rip }}}-1 \leq 0, \text { with } k=\mathrm{LC} 1, \mathrm{LC} 2,
$$

where $\delta_{\text {tip }}^{\text {ref }}=0.2 b$ and $b=17000 \mathrm{~mm}$ is the semi-span of the wing, while $\delta_{\text {tip }}$ is the vertical displacement at the center of the rib located at the wing tip.

Similarly, OC2 (which is related to DC2) is formulated by considering the twist of the wing tip rib in order to impose a sufficient torsional stiffness to the structure:

$$
h_{2}:=\max _{k} \frac{\left|\theta_{\text {tip }}\right|}{\theta_{\text {tip }}^{\text {rip }}}-1 \leq 0, \text { with } k=\mathrm{LC} 1, \mathrm{LC} 2,
$$

where $\theta_{\text {tip }}$ is the rotation around the wing span axis and $\theta_{\text {tip }}^{\text {ref }}=6$ deg.

OC3 takes into account for the fatigue failure criterion of DC3, which is expressed as an equivalent check on the longitudinal stress $\sigma_{l}$ resulting from the static analysis (for each LC), i.e.

$$
h_{3}:=\max _{k} \frac{\left|\sigma_{l}\right|}{\sigma_{\max }}-1 \leq 0, \text { with } k=\mathrm{LC} 1, \mathrm{LC} 2, \text { in } \Gamma_{\text {check }},
$$

where $\sigma_{\max }=250 \mathrm{MPa}$ and $\sigma_{l}$ is the longitudinal maximum value of stress resulting from the GFEM. The calculation of $\sigma_{l}$ is anything but trivial: it is assessed by considering all the RSUs belonging to $\Gamma_{\text {check }}$ and by computing the resulting total longitudinal force as the sum of the one extracted from the beam element (stringer) and the half of the ones extracted from the adjacent shell elements (collaborating skin). Accordingly, $\sigma_{l}$ is obtained by dividing the RSU total force by the RSU section area, which changes depending on the position inside $\Gamma_{\text {check }}$.

The fourth optimization constraint (OC4) formalizes the no-buckling requirement of DC4. To this end, an eigenvalue buckling analysis is performed on the most critical stiffened panels constituting the ZOIs extracted from $\Gamma_{\text {check }}$. This operation is carried out by means of an on-line generation of a refined LFEM of the ZOI for which the buckling analysis is performed. In order to enforce consistent boundary conditions to the LFEM of the ZOI, a sub-modelling approach is used (more details are provided in Sec. 4.1). OC4 is formulated as follows:

$$
h_{4}:=\max _{k}\left(1-\frac{\lambda}{\lambda_{\text {cr }}}\right) \leq 0, \text { with } k=\mathrm{LC} 1, \mathrm{LC} 2, \text { in } \Gamma_{\mathrm{LFEM}},
$$

where $\lambda$ is the buckling factor resulting from the LFEM and $\lambda_{\mathrm{cr}}=1.6$.

OC5 makes use of a simplified analytical approach [5] to establish the minimum allowable rib thickness, $t_{\text {rib-cr }}$ which prevents rib from warping under compressive load. The constraint is expressed as follows:

$$
\begin{gathered}
h_{5}:=\max _{k} \\
t_{\text {rib-cr }}= \\
\pi^{4} E L h \rho_{c}
\end{gathered} \quad\left(1-\frac{t_{\text {rib }}}{t_{\text {rib-cr }}}\right) \leq 0, \quad \text { with } k=\mathrm{LC} 1, \mathrm{LC} 2,
$$


In Eq. (12) $t_{\text {rib }}$ is the thickness of the rib, whilst $w, h$ and $\rho_{c}$ are the width, the height and the radius of gyration of the rib, respectively, $E$ is the Young's modulus of the material of the rib, $L$ is the rib pitch and $N_{\text {cr }}$ is the critical load of the repetitive unit.

Finally, the CNLPP is formulated as follows:

$$
\begin{gathered}
\min _{\boldsymbol{\xi}} \Phi(\xi), \\
\text { subject to } h_{i}(\boldsymbol{\xi}) \leq 0, i=1, \ldots, 5
\end{gathered}
$$

where lower and upper bounds of $\boldsymbol{\xi}$ provided in Table 3 .

\subsection{Numerical strategy}

The ERASMUS (EvolutionaRy Algorithm for optimiSation of ModUlar Systems) optimization tool, coupled with the ANSYS FE commercial software, is used to perform the solution search of problem (13). The genetic algorithm (GA) ERASMUS has already successfully been used to solve real-world engineering problems of different nature, see for example [25, 29, 31, 32, 34].

Problem 13 is a non-convex CNLPP with 29 design variables and 5 optimization constraints. The overall architecture of the numerical procedure is illustrated in Fig. 10. The numerical process can be divided into two parts: the GA operations and the FE solution. The ERASMUS algorithm performs the genetic operations which allows the selection of the fittest individuals on the basis of the values of both the objective function, $\Phi$, and the optimization constraints, $h_{i}$. These are calculated from the physical responses provided by both GFEM and LFEM.

The generation of the FE models (GFEM and LFEM) is performed through a fully para-

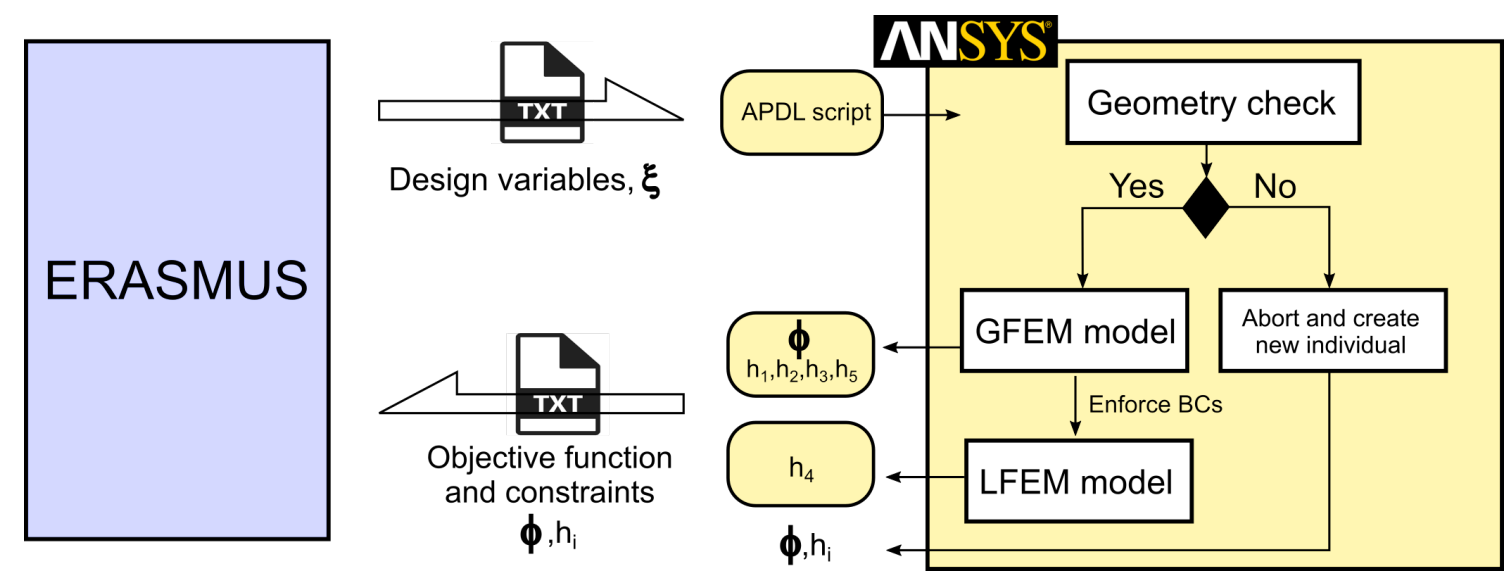

Figure 10: Global architecture of the numerical strategy for problem (13).

metric Ansys Parametric Design Language (APDL) script which reads the design variables returned by the ERASMUS code. Before generating the GFEM model a check is performed on the value of the design variables of each individual to ensure non-decreasing values of the geometrical parameters defining the stringer section along the wing span. In particular, both $\chi_{\mathrm{str}}^{\text {top }}$ and $\chi_{\mathrm{str}}^{\text {bot }}$ are checked in order to obtain $\alpha_{\mathrm{in}} \geq 1$ and $\alpha_{\text {out }} \geq 1$, see Eq. (4). Whenever these conditions are not fulfilled, the APDL script does not create the GFEM and returns penalized values of $\Phi$ and $h_{i}$ to ERASMUS.

The generic individual of the GA, which represents a potential solution of the non-convex 
CNLPP, has a genotype characterized by one chromosome containing 29 genes corresponding to the components of the vector of the design variables $\boldsymbol{\xi}$.

\section{The Global/Local Finite Element Modelling Approach}

As stated in Sec. 3.3, the optimization strategy makes use of two FE models, the GFEM and the LFEM, to assess the set of DCs. GFEM and LFEM are characterized by different features, which aim at giving a proper evaluation of both global and local structure responses, respectively.

The GL FE approach is characterized by the following steps:

a. generation of the parametric GFEM of the wing-box in terms of the design variables provided by ERASMUS;

b. static analysis of the GFEM, for each LC, and evaluation of the objective function and GFEM responses (involved in the optimization constraints definition);

c. identification of two ZOIs (top and bottom regions of the wing), generation of two LFEM, application of the interpolated displacement at the boundary of the ZOI and evaluation of the corresponding buckling factors.

d. generation of the output file with objective function and optimization constraints for ERASMUS.

\subsection{The Global Finite Element Model}

The macroscopic behaviour of the wing-box is simulated by means of 4-node SHELL181 elements with four nodes and six degrees of freedom (DOFs) per node (Reissner-Mindlin theory) for skin, ribs and spar webs and 2-node BEAM188 elements with two nodes and six DOFs per node (Timonshenko's beam theory) for stringers and spar caps. At the intersections, beam and shell elements are connected through node merging and, to correctly model the position of stringers with respect to the skin, an offset is defined and applied to the beam cross-section. Ribs are directly connected to the skin by merging the nodes at the intersections, which ensures the transfer of the shear loads between the two structural elements. A sensitivity analysis on the mesh size has been carried out, but it is not be reported here for the sake of brevity. The total number of elements of the GFEM is approximately 6600. The mesh of shell elements is mainly composed of quadrangular elements with triangular elements occurring close to the runout areas, see Fig. 11.

Aerodynamic forces and moments as well as the loads due to the engine weight are applied to reference nodes, created at the centroid of each rib, which are linked to the nodes at the boundary of each rib. The connection is ensured via RBE3 elements as shown in Fig. 12. Eventually, all DOFs belonging to the wing-box root section have been set equal to 0 to simulate a fully clamped BC.

The results of the analyses carried out on the GFEM, for both LCs, provide the objective function and the values of four OCs: $h_{1}, h_{2}, h_{3}$ and $h_{5}$. The value of the remaining OC, $h_{4}$, is obtained by means of the LFEM.

\subsection{The Local Finite Element Model}

The objective of the LFEM is to capture mechanical phenomena, at the scale of the structural component, which would not appear at the global wing-box scale and that, thus, cannot be assessed through the analyses conducted on the GFEM. The aim of the LFEM is to compute the first buckling load of the most critical stiffened panel.

The LFEM is generated for the ZOI identified within $\Gamma_{\text {check }}$, see Fig. 9 . $\Gamma_{\text {check }}$ is built by excluding the following regions from the GFEM: 


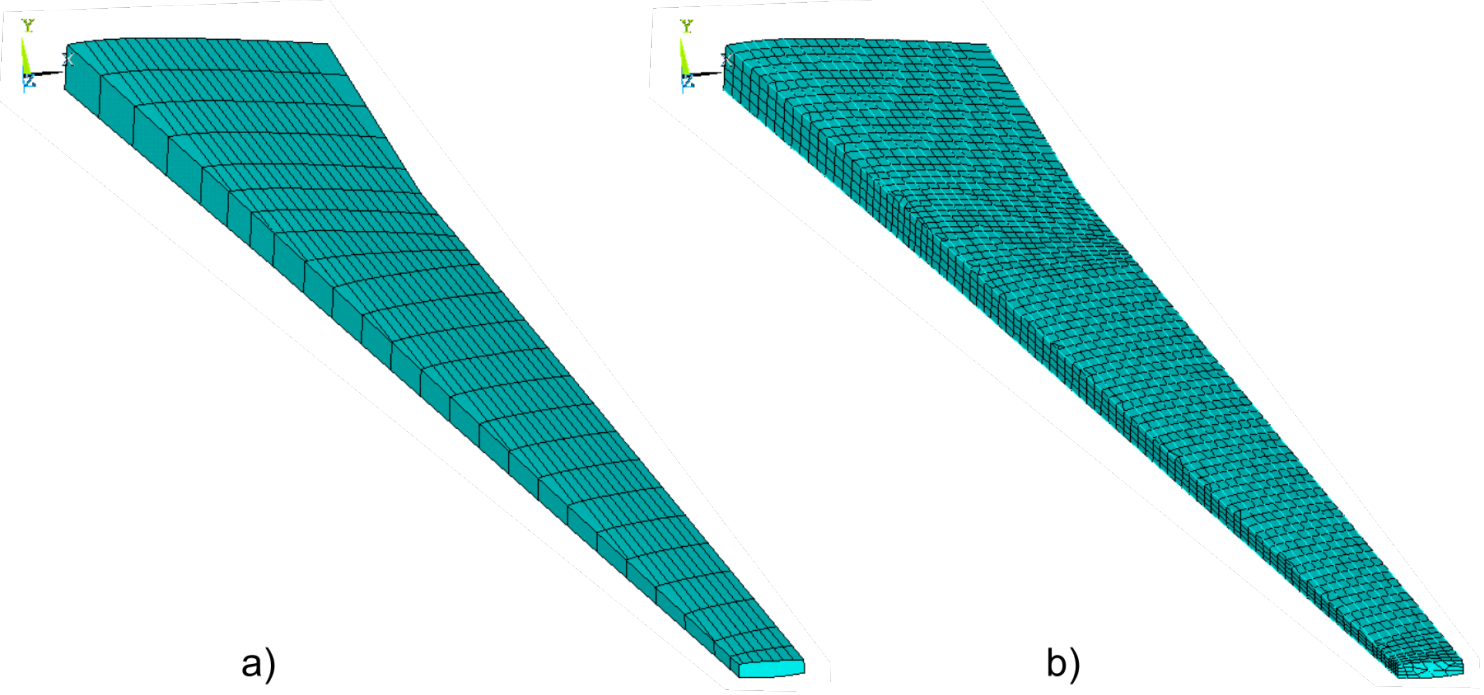

Figure 11: Example of the GFEM geometry (a) and of the mesh used in the GFEM analyses (b).

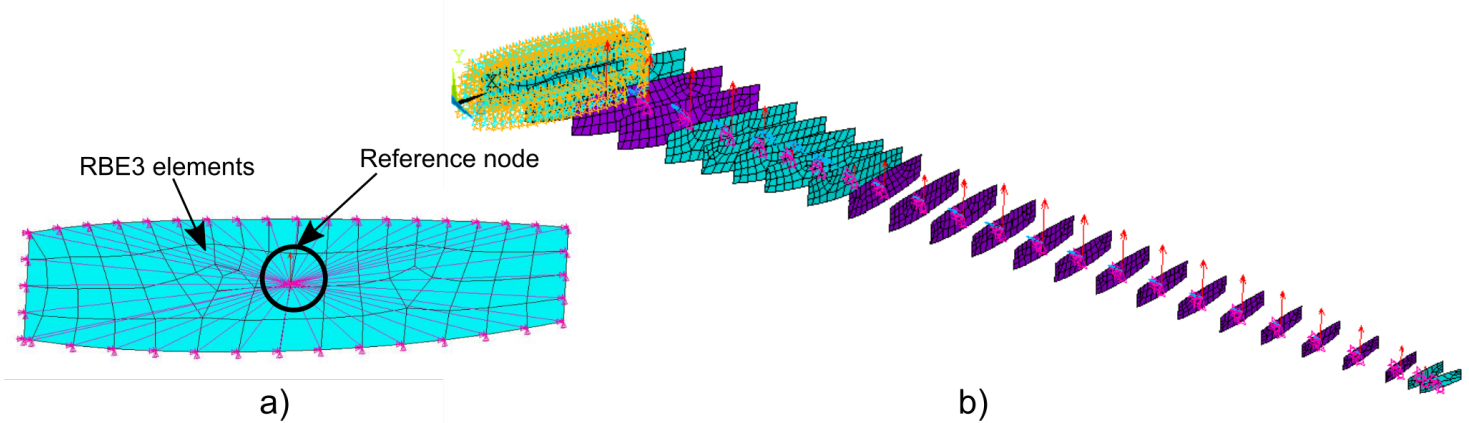

Figure 12: (a) Reference node and RBE3 elements created for a rib. (b) Application of forces and moments to the ribs.

- the first three bays adjacent to the wing-box root section are excluded;

- transition bays are excluded;

- the first two stringers adjacent to the front spar and the rear one are excluded;

- the last stringer segment before runout takes place is excluded;

- bays with less than five stringers are excluded.

Thanks to the parametric scripts, the user can choose a priori the number of stringers to be included within the LFEM. In this study, this number has been set equal to five, as shown in Fig. 13 (a). The mesh has been adapted to have at least three elements to discretize flanges, webs and at least four elements between stringers. The aspect ratio of the elements is checked in order to be lower than or equal to two.

The criterion used to evaluate the ZOI is based on the load index, $N_{\mathrm{RSU}}$, computed for every RSU [39]. By analysing the GFEM results on $\Gamma_{\text {check }}$, a map of the values of $N_{i}$, for the $i$-th RSU of $\Gamma_{\text {check }}$ is obtained and the one characterized by the highest $N_{\mathrm{RSU}}$ is 
selected. In particular, for the $i$-th RSU, $N_{i}$ is computed as:

$$
N_{i}:=N_{11}^{\mathrm{str}}+\frac{N_{11}^{\mathrm{skin}_{j}}}{2}+\frac{N_{11}^{\mathrm{skin}_{j+1}}}{2},
$$

where $N_{11}^{\text {str }}$ is the stringer load, extracted from the beam elements of the GFEM, in the $x_{1}$ direction of Fig. 13 (a), $N_{11}^{\text {skin }_{j}}$ and $N_{11}^{\text {skin }_{j+1}}$ are the membrane loads (per unit of width) in the $x_{1}$ direction of the portions of skin adjacent to the stringer.

Once the ZOI is identified, the creation of the LFEM is carried out through the following steps:

a. Starting from the ZOI location, the GFEM is partitioned in order to reproduce the geometry of Fig. 13 (a). The outer geometry and the associated mesh is deleted.

b. The relevant geometrical properties of the beam cross-section are extracted and an ad-hoc APDL script creates the corresponding stringers modelled with shell elements (SHELL181), as shown in Fig. 13 (b).

c. Stringer flanges are tied to the skin with MPC184 elements which creates rigid connections between facing nodes.

d. At the LFEM boundary, stringer cross-sections are tied via RBE3 elements to reference nodes of the skin as shown in Fig. 13 (b).

e. BCs, taken from the GFEM results, are enforced on the external boundary of the LFEM as well as on the segments resulting from the intersection of the two ribs (present in the GFEM) with the skin geometry where the skin regions facing the stringer flanges are excluded.

Subsequently, a linear buckling analysis is performed on the critical LFEM from which the first positive eigenvalue, $\lambda(\boldsymbol{\xi})$, is used to evaluate the OC $h_{4}$.

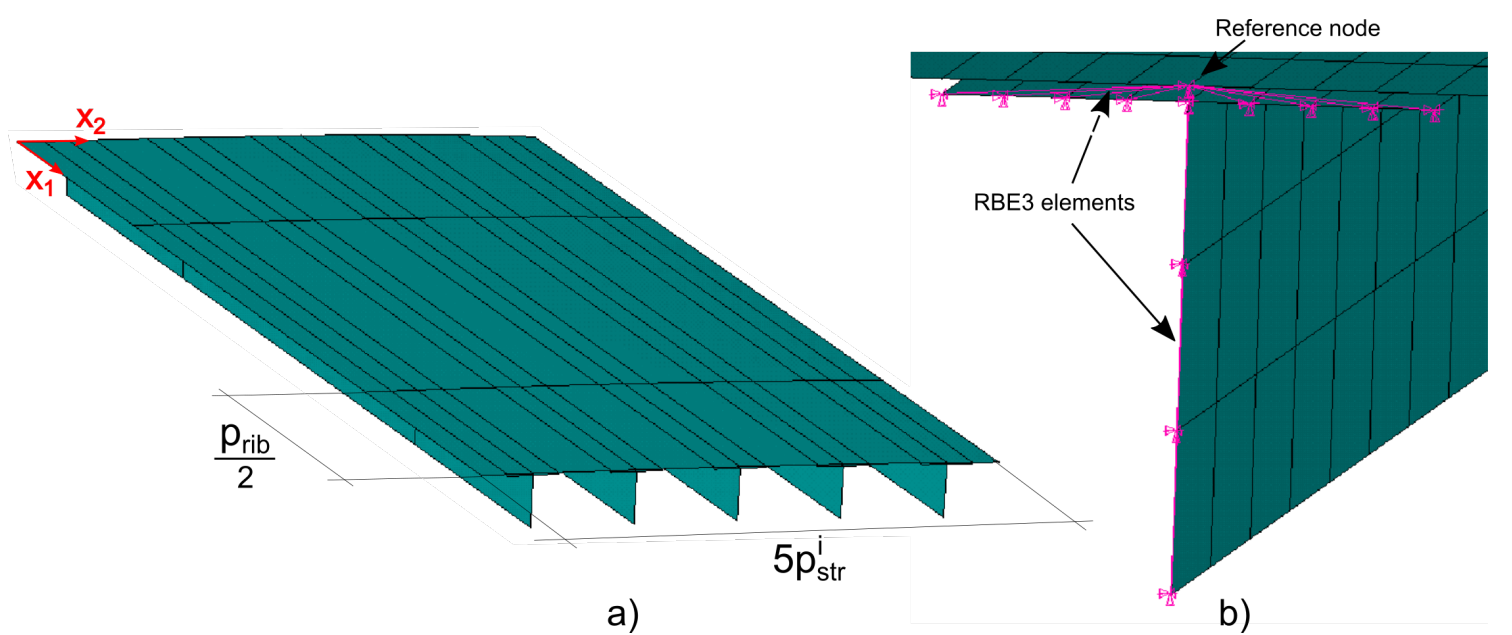

Figure 13: (a) Dimensions of the 5-stringer LFEM where $p_{\text {rib }}=700 \mathrm{~mm}$. (b) Detail of the shell geometry generated in the LFEM from the geometrical properties of the beam element sections in the GFEM.

\section{Numerical results}

To solve optimization problem (13), the parameters governing the behaviour of the ERASMUS algorithm are listed in Table 4. As far as unfeasible individuals are con- 
cerned, optimization constraints are handled via the Automatic Dynamic Penalization (ADP) method is used [40]. The selection of the individual for the reproduction phase is carried out through the roulette-wheel operator [25]. The computational effort required to complete the whole optimization process is of approximately 10 days using two cores of an Intel Xeon E5-2697v2 processor (2.70-3.50 GHz). In Fig. 14, the objective function history (wing-box mass) for each generation is shown. After 80 generations, the overall mass of the wing reduces approximately to $1557 \mathrm{~kg}$. Table 5 reports the value of the objective function as well as the values of the OCs. As it can been inferred from these results, the most critical OC is that related to the maximum longitudinal stress, see Eq. (10), and the one on the minimum thickness of the ribs. Conversely, the OCs on the buckling factor, on the vertical displacement and on the rotational stiffness are not active (and thus not critical) for the optimised configuration.

The values of the design variables characterizing the optimized solution of problem (13) are provided in Table 6 . All the design variables of the optimal individual lie within the bounds defined in Table 3 . The pitch of the stringers in top and bottom regions of the wing are very close, but the stringers cross-section parameters characterizing the top region are significantly greater than the counterparts characterizing the bottom region.

Fig. 15 shows, for the optimal individual of problem (13), the vertical displacement of the GFEM, the buckling mode shape and the corresponding buckling factor of the LFEM built to evaluate the OC $h_{4}$. The buckling mode shape is consistent with the instabilities typically observable on such structures. In particular, a combined instability of both skin and stringers is obtained, which is in agreement with the theory [39]. The value obtained for the buckling factor, as reported in Table 5 , highlights that, with respect to the OC $h_{4}$, a margin of improvement of approximately $15 \%$ still exists.

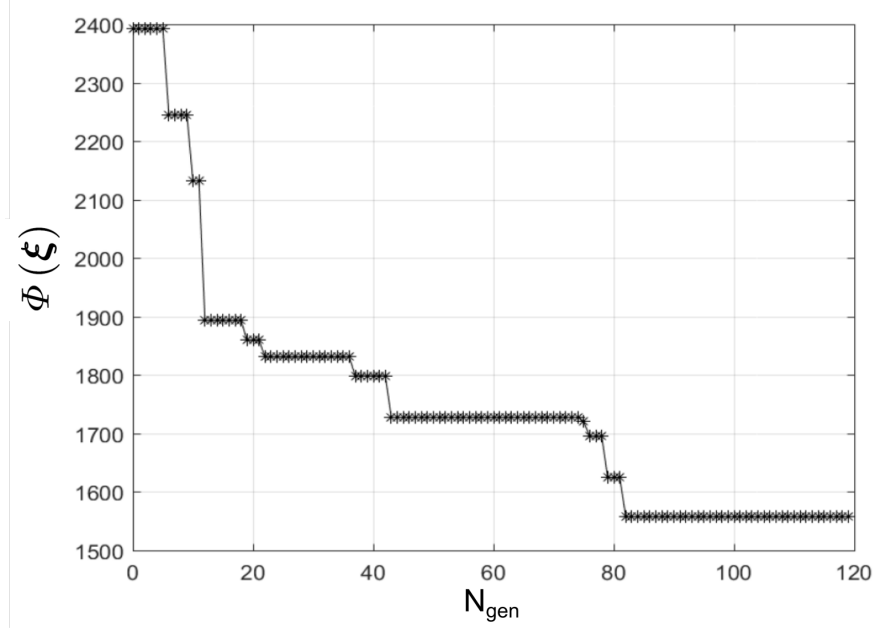

Figure 14: Evolution of the wing-box structural mass, $\Phi(\boldsymbol{\xi})$ vs. the generation number $\left(N_{\text {gen }}\right)$

\section{Conclusions}

The design strategy presented in this work is based on a multi-scale method, which makes use of a global/local modelling approach. The least-weight design of a wing-box structure subject to multiple loading conditions and to several design requirements has been taken as a real-life engineering application to show the effectiveness of the proposed 


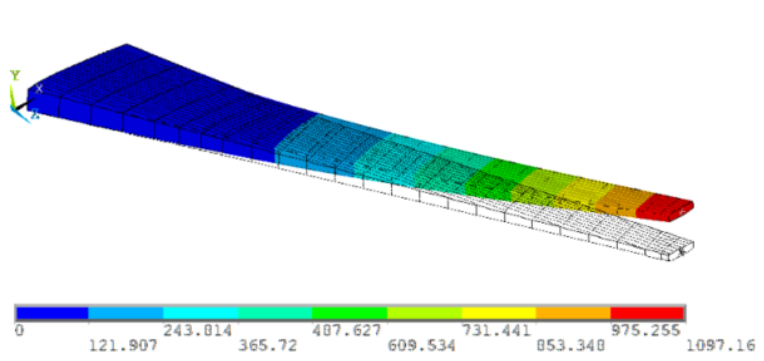

a)

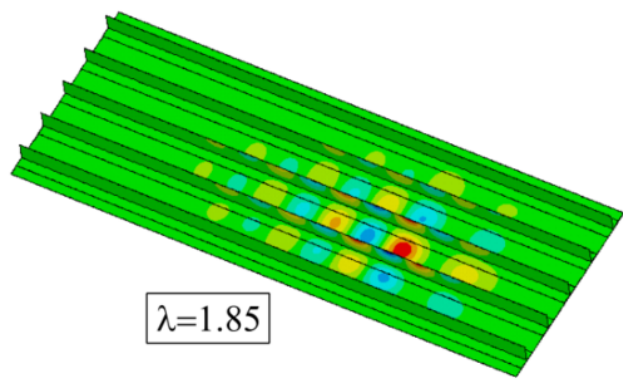

b)

Figure 15: Optimized configuration of the wing-box (load case LC1): (a) vertical displacement from the GFEM, (b) buckling mode shape and buckling factor of the LFEM.

multi-scale optimization strategy. The design problem is formulated as a constrained nonlinear programming problem, where different mechanical geometrical and manufacturing requirements are integrated. The structural response is assessed by considering design requirements at different scales. To this purpose, at the global scale (i.e. the one of the whole wing-box architecture) a low-fidelity finite element model is built to assess requirements on the overall stiffness of the structure (in terms of displacements and rotations). Conversely, an high-fidelity finite element model is automatically generated for the most critical zones of the global model.

The generation of the local finite element model is performed without the user's intervention and it is driven by ad hoc criteria applied to the results of the global finite element model. A dedicated procedure has also been developed to transfer the real (and not idealized) boundary conditions from the results of the global finite element model to the most critical regions, where the local finite element model is generated. This task is anything but trivial because low-fidelity and high-fidelity models are not characterized by the same element type and the same mesh. To perform the solution search for this complicate multi-scale problem, the ERASMUS algorithm, developed at the I2M laboratory, has been succesfully used.

As far as the prospects of this work are concerned, research is ongoing in order to generalise the proposed multi-scale optimization strategy, which integrates a global/local modelling approach, to the case of composite thin-walled structures. Moreover, about the real-world engineering problem discussed in this work, the effectiveness of the proposed approach needs to be tested by integrating further loading cases dealing with non-symmetric manoeuvrers, dynamic and static gusts and aeroelastic design requirements. Nonetheless, due to the nature of the ERASMUS algorithm, several feasible individuals are available within the population at each generation. These feasible individuals, which are not optimal with respect to the problem at hand, can be collected in a sort of "database of feasible solutions", which can be used in the post-processing phase to check solutions complying with further design criteria (e.g. aeroelasticity, gusts and non-symmetric manoeuvres). Therefore, the result of the use of a meta-heuristic algorithm is not only the determination of an optimal individual, but also the generation of a database of feasible solutions to be exploited for different purposes related to the nature of the problem at hand. Finally, a further interesting prospect is about the development of a pertinent problem formulation, integrating a 
generalised version of the global/local modelling approach, compatible with a deterministic optimization algorithm. The main challenge in this case is to develop suitable aggregation techniques and continuation methods in order to derive the form of the gradient of the responses evaluated on the local model and to deal with the discontinuous behaviour of the check zone (which could occur at different locations of the global model depending on the current value of the design variables). Research is ongoing on the above aspects.

\section{Acknowledgements}

This paper presents part of the activities carried out within the research project PARSIFAL (Prandtlplane ARchitecture for the Sustainable Improvement of Future AirpLanes), which has been funded by the European Union under the Horizon 2020 Research and Innovation Program (Grant Agreement n. 723149).

\section{References}

[1] J. Wignot, H. Combs, A. Ensrud, Analysis of circular shell-supported frames, Technical note 929, NACA (1944).

[2] P. Kuhn, J. Peterson, L. Levin, Summary of diagonal tension, Technical note 2661, NACA (1952).

[3] G. Gerard, The Crippling Strength of Compression Elements, Journal of the Aerospace Sciences 25 (1) (1958) 37-52. doi:10.2514/8.7482.

[4] M. Niu, Airframe structural design: practical design information and data on aircraft structures, Conmilit Pr, 1988.

[5] E. Bruhn, Analysis and design of flight vehicle structures, Tri-State Offset Co., 1973.

[6] S. Grihon, M. Samuelides, A. Merval, A. Remouchamps, M. Bruyneel, B. Colson, K. Hertel, Fuselage structure optimisation, in: Advances in collaborative civil aeronautical multidisciplinary design optimization, Progress in astronautics and aeronautics, AIAA, 2009. doi:10.2514/4.867279.

[7] O. Hughes, B. Ghosh, Y. Chen, Improved prediction of simultaneous local and overall buckling of stiffened panels, Thin-Walled Structures 42 (6) (2004) $827-856$.

[8] D. Stamatelos, G. Labeas, K. Tserpes, Analytical calculation of local buckling and post-buckling behavior of isotropic and orthotropic stiffened panels, Thin-Walled Structures 49 (3) (2011) $422-430$.

[9] J. Sobieszczanski, D. Loendorf, A Mixed Optimization Method for Automated Design of Fuselage Structures, Journal of Aircraft 9 (12) (1972) 805-811.

[10] M. Fischer, D. Kennedy, C. Featherston, Multilevel framework for optimization of lightweight structures, Proceedings of the Institution of Mechanical Engineers, Part G: Journal of Aerospace Engineering 226 (4) (2012) 380-394.

[11] S. Venkataraman, R. Haftka, Structural optimization complexity: what has moore's law done for us?, Structural and Multidisciplinary Optimization 28 (6) (2004) 375-387.

[12] I. Hirai, B. Wang, W. Pilkey, An efficient zooming method for finite element analysis, International Journal for Numerical Methods in Engineering 20 (9) (1984) 1671-1683. 
[13] C. Sun, K. Mao, A global-local finite element method suitable for parallel computations, Computers \& Structures 29 (2) (1988) 309-315.

[14] K. Mao, C. Sun, A refined global-local finite element analysis method, International Journal for Numerical Methods in Engineering 32 (1) (1991) 29-43.

[15] J. Whitcomb, Iterative global/local finite element analysis, Computers \& Structures 40 (4) (1991) 1027-1031.

[16] N. Cormier, B. Smallwood, G. Sinclair, G. Meda, Aggressive submodelling of stress concentrations, International Journal for Numerical Methods in Engineering 46 (6) (1999) 889-909.

[17] O. Dababneha, T. Kipouros, A review of aircraft wing mass estimation methods, Aerospace Science and Technology 72 (2018) 256-266.

[18] A. Benaouali, S. Kachel, Multidisciplinary design optimization of aircraft wing using commercial software integration, Aerospace Science and Technology 92 (2019) 766 776 .

[19] A. Zhao, H. Zou, H. Jin, D. Wen, Structural design and verification of an innovative whole adaptive variable camber wing, Aerospace Science and Technology 89 (2019) 11 -18 .

[20] O. Dababneha, T. Kipouros, Influence of high fidelity structural models on the predicted mass of aircraft wing using design optimization, Aerospace Science and Technology 79 (2018) 164-173.

[21] A. Arrieta, A. G. Stritz, Optimal design of aircraft structures with damage tolerance requirements, Struct Multidisc Optim. 30 (2005) 155-163.

[22] P. Ciampa, B. Nagel, M. Tooren, Global local structural optimization of transportation aircraft wings, in: 51st AIAA/ASME/ASCE/AHS/ASC Structures, Structural Dynamics, and Materials Conference, 2010.

[23] V. Chedrik, Two-level design optimization of aircraft structures under stress, buckling and aeroelasticity constraints, in: 10th World Congress on Structural and Multidisciplinary Optimisation, 2013.

[24] Q. Liu, J. Mohamed, B. M. Sameer, K. K. Rakesh, Integrated global wing and local panel optimization of aircraft wing, in: 56th AIAA/ASCE/AHS/ASC Structures, Structural Dynamics, and Materials Conference, 2015.

[25] M. Montemurro, A contribution to the development of design strategies for the optimisation of lightweight structures, Hdr thesis, Universite de Bordeaux (2018).

URL http://hdl.handle.net/10985/15155

[26] M. Montemurro, A. Vincenti, P. Vannucci, A two-level procedure for the global optimum design of composite modular structures - application to the design of an aircraft wing. part 1: theoretical formulation, Journal of Optimization Theory and Applications 155 (1) (2012) 1 - 23. 
[27] M. Montemurro, A. Vincenti, P. Vannucci, A two-level procedure for the global optimum design of composite modular structures - application to the design of an aircraft wing. part 2: numerical aspects and examples, Journal of Optimization Theory and Applications 155 (1) (2012) $24-53$.

[28] M. Montemurro, A. Catapano, D. Doroszewski, A multi-scale approach for the simultaneous shape and material optimisation of sandwich panels with cellular core, Composites Part B: Engineering 91 (2016) 458 - 472.

[29] M. Montemurro, A. Catapano, A new paradigm for the optimum design of variable angle tow laminates, in: G. Buttazzo, A. Frediani (Eds.), Variational analysis and aerospace engineering: mathematical challenges for the aerospace of the future, Vol. 116 of Springer Optimization and Its Applications, Springer, New York, NY, 2016. doi: $10.1007 / 978-3-319-45680-5$.

[30] G. Costa, M. Montemurro, J. Pailhès, A general hybrid optimization strategy for curve fitting in the non-uniform rational basis spline framework, Journal of Optimization Theory and Applications 176 (1) (2018) 225 - 251.

[31] M. Montemurro, A. Catapano, On the effective integration of manufacturability constraints within the multi-scale methodology for designing variable angle-tow laminates, Composite Structures 161 (2017) 145 - 159.

[32] M. Montemurro, M. I. Izzi, J. E. Yagoubi, D. Fanteria, Least-weight composite plates with unconventional stacking sequences: Design, analysis and experiments, Journal of Composite Materials 53 (16) (2019) 2209-2227.

[33] E. Panettieri, M. Montemurro, A. Catapano, Blending constraints for composite laminates in polar parameters space, Composites Part B: Engineering 168 (2019) 448-457.

[34] M. Montemurro, A. Pagani, G. A. Fiordilino, J. Pailhès, E. Carrera, A general multiscale two-level optimisation strategy for designing composite stiffened panels, Composite Structures 201 (2018) $968-979$.

[35] Airbus A320 aircraft characteristics for airport and maintenance planning, Tech. rep., AIRBUS S.A.S., Customer Services Technical Data Support and Services (dowloaded from https://www.airbus.com/aircraft/support-services/airportoperations-and-technical-data/aircraft-characteristics.html) (2010).

[36] D. P. Raymer, Aircraft design: a conceptual approach, Educ Series, American Institute of Aeronautics and Astronautics, 1989.

[37] U. G. Goranson, Fatigue issues in aircraft maintenance and repairs, International Journal of Fatigue 20 (6) (1998) 413-431.

[38] Technical note 2751, National Advisiory Committee for Aeronautics (1951).

[39] G. Gerard, Minimum weight analysis of compression structures, New York University Press, 1956.

[40] M. Montemurro, A. Vincenti, P. Vannucci, The Automatic Dynamic Penalisation method (ADP) for handling constraints with genetic algorithms, Computer Methods in Applied Mechanics and Engineering 256 (2013) 70-87. 
Table 3: Design variables, lower and upper bounds and discretization step $(i=$ top, bottom and $j=$ in, out).

\begin{tabular}{|c|c|c|c|}
\hline Design variable & Lower bound & Upper bound & Discretization step \\
\hline \multicolumn{4}{|c|}{ Skin } \\
\hline$t_{\text {skin-tip }}^{i}[\mathrm{~mm}]$ & 2.7 & 5.5 & 0.05 \\
\hline$\alpha_{\text {skin }-j}^{i}[-]$ & 1 & 4 & 0.05 \\
\hline \multicolumn{4}{|c|}{ Spar } \\
\hline$t_{\text {spar-tip }}[\mathrm{mm}]$ & 1.2 & 4 & 0.05 \\
\hline$\alpha_{\text {spar }-j}[-]$ & 1 & 4 & 0.05 \\
\hline \multicolumn{4}{|c|}{ Rib } \\
\hline$t_{\text {rib-tip }}[\mathrm{mm}]$ & 1.2 & 4 & 0.05 \\
\hline$\alpha_{\mathrm{rib}-j}[-]$ & 1 & 4 & 0.05 \\
\hline \multicolumn{4}{|c|}{ Spar cap } \\
\hline$w_{\text {spar-tip }}[\mathrm{mm}]$ & 16 & 24 & 0.05 \\
\hline$t_{1-\text { spar-tip }}[\mathrm{mm}]$ & 2.1 & 6 & 0.05 \\
\hline$t_{2-\text { spar-tip }}[m m]$ & 1.2 & 4 & 0.05 \\
\hline$\alpha_{\text {spar }-j}[-]$ & 1 & 4 & 0.05 \\
\hline \multicolumn{4}{|c|}{ Stringer } \\
\hline$p_{\mathrm{str}}^{i}[\mathrm{~mm}]$ & 100 & 160 & 1 \\
\hline$w_{1-\operatorname{str}-\operatorname{tip}}^{i}[m m]$ & 32 & 80 & 0.05 \\
\hline$w_{1-\operatorname{str}-\operatorname{root}}^{i}[m m]$ & 32 & 80 & 0.05 \\
\hline$t_{1-\operatorname{str}}^{i}[m m]$ & 1.2 & 5 & 0.05 \\
\hline$t_{2-\operatorname{str}}^{i}[m m]$ & 1.2 & 5 & 0.05 \\
\hline$\chi_{\mathrm{str}}^{i}[-]$ & 0.1 & 1.1 & 0.05 \\
\hline
\end{tabular}


Table 4: Parameters of the GA ERASMUS used for the optimization.

\begin{tabular}{ccc}
\hline Parameter & Description & Value \\
\hline$N_{\text {pop }}$ & Number of populations & 2 \\
\hline$N_{\text {ind }}$ & Number of individuals per population & 290 \\
\hline$N_{\text {gen }}^{\max }$ & Max. number of generations & 120 \\
\hline$p_{\text {cross }}$ & Crossover probability & 0.85 \\
\hline$p_{\text {mut }}$ & Crossover probability & $1 / N_{\text {ind }}$ \\
\hline
\end{tabular}

Table 5: Objective function and values of the OCs for the optimal individual.

\begin{tabular}{ccccccc}
\hline Function & $\Phi[\mathrm{kg}]$ & $h_{1}$ & $h_{2}$ & $h_{3}$ & $h_{4}$ & $h_{5}$ \\
Value & 1557.5 & -0.5768 & -0.6582 & -0.002 & -0.1494 & -0.001 \\
\hline
\end{tabular}

Table 6: Design variables of the optimal individual solution of problem (13).

\begin{tabular}{|c|c|c|c|c|c|c|c|c|}
\hline \multirow[t]{2}{*}{ Design variable } & \multicolumn{2}{|c|}{ Top } & \multicolumn{2}{|c|}{ Bottom } & \multirow[t]{2}{*}{ Design variable } & \multicolumn{2}{|c|}{ Top } & Bottom \\
\hline & & Out & In & Out & & In & Out & In Out \\
\hline$t_{\text {skin-tip }}^{i}[\mathrm{~mm}]$ & \multicolumn{2}{|c|}{4.7} & \multicolumn{2}{|c|}{4.3} & $t_{2-\text { spar-tip }}[\mathrm{mm}]$ & \multicolumn{3}{|c|}{1.4} \\
\hline$\alpha_{\mathrm{skin}-j}^{i}[-]$ & 1.2 & 1.1 & 1.4 & 2.7 & $\alpha_{\text {spar-j }}[-]$ & 2.7 & 1.1 & \multirow[b]{2}{*}{145.1} \\
\hline$t_{\text {spar-tip }}[\mathrm{mm}]$ & \multicolumn{4}{|c|}{1.3} & $p_{\mathrm{str}}^{i}[\mathrm{~mm}]$ & \multicolumn{2}{|c|}{144.7} & \\
\hline$\alpha_{\mathrm{spar}-j}[-]$ & \multicolumn{4}{|c|}{2.6} & $w_{1-\text { str-tip }}^{i}[\mathrm{~mm}]$ & \multicolumn{2}{|c|}{51.7} & 33.9 \\
\hline$t_{\text {rib-tip }}[\mathrm{mm}]$ & \multicolumn{4}{|c|}{1.4} & $w_{1-\operatorname{str}-\operatorname{root}}^{i}[\mathrm{~mm}]$ & \multicolumn{2}{|c|}{72} & 36.7 \\
\hline$\alpha_{\mathrm{rib}-j}[-]$ & \multicolumn{4}{|c|}{1.1} & $t_{1-\mathrm{str}}^{i}[\mathrm{~mm}]$ & \multicolumn{2}{|c|}{1.8} & 1.7 \\
\hline$w_{\text {spar-tip }}[\mathrm{mm}]$ & \multicolumn{4}{|c|}{17.9} & $t_{2-\text { str }}^{i}[\mathrm{~mm}]$ & \multicolumn{2}{|c|}{4.0} & 1.9 \\
\hline$t_{1-\text { spar-tip }}[\mathrm{mm}]$ & \multicolumn{4}{|c|}{4.7} & $\chi_{\mathrm{str}}^{i}[-]$ & \multicolumn{2}{|c|}{1.05} & 1.10 \\
\hline
\end{tabular}

